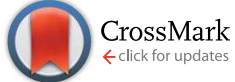

Cite this: J. Mater. Chem. A, 2015, 3, 12730

Received 8th February 2015

Accepted 8th May 2015

DOI: $10.1039 / c 5 t a 01036 e$

www.rsc.org/MaterialsA

\section{Enhancement of capacitive deionization capacity of hierarchical porous carbon $\uparrow$}

\author{
Lumeng Chao, ${ }^{\text {a }}$ Zhenyu Liu, ${ }^{\text {ac }}$ Guoxin Zhang, ${ }^{a}$ Xiaona Song, ${ }^{a}$ Xiaodong Lei, ${ }^{a}$ \\ Michael Noyong, ${ }^{\text {b }}$ Ulrich Simon, ${ }^{\text {b }}$ Zheng Chang ${ }^{\star a}$ and Xiaoming Sun ${ }^{\star a}$
}

\begin{abstract}
Capacitive deionization (CDI) has attracted huge interest as an energy-efficient and eco-friendly desalination strategy. Its development is presently limited due to the relatively low CDI capacitances of carbon materials. Herein, hierarchical porous carbon materials (HPCs) derived from ethylenediaminetetraacetic acid (EDTA) upon annealing were used, which showed impressive CDI performance with a maximum desalination capacity of $34.27 \mathrm{mg} \mathrm{g}^{-1}$ in $40 \mathrm{mg} \mathrm{L}^{-1} \mathrm{NaCl}$ aqueous solution. Such capability was attributed to the appropriate hierarchical pore structure, high specific surface area $\left(2185.71 \mathrm{~m}^{2} \mathrm{~g}^{-1}\right)$, large pore volume $\left(1.368 \mathrm{~cm}^{3} \mathrm{~g}^{-1}\right)$ and reasonable graphitization degree, which were also confirmed by the high specific capacitances of $182 \mathrm{~F} \mathrm{~g}^{-1}$ in $1 \mathrm{~mol} \mathrm{~L}^{-1} \mathrm{NaCl}$ and $260 \mathrm{~F} \mathrm{~g}^{-1}$ in $6 \mathrm{~mol} \mathrm{~L}^{-1} \mathrm{KOH}$. Since the physisorption capacity was nearly 0 , and the regeneration process was facile and complete, such economical HPCs materials show potential for practical desalination applications in the future. Moreover, the HPCs electrodes presented ion selectivity in competitive multi-ionic solutions by kinetic behavior difference or static capacitance difference.
\end{abstract}

\section{Introduction}

Water shortage has evolved to be one of the most challenging obstacles lying in front of the ever increasing economy and population, and desalination of sea water has been considered promising for relieving the water shortage crisis. ${ }^{\mathbf{1} 2}$ Recently, capacitive deionization (CDI) has been regarded as one of the most applicable methods ${ }^{3,4}$ among current available strategies for desalination, i.e. reverse osmosis, ${ }^{5}$ electrodialysis, ${ }^{6}$ distillation $^{7}$ and others. The advantages of CDI are its low applied voltage $(<2 \mathrm{~V})$, inexpensiveness, avoidance of secondary wastes, and easiness of device or plant regeneration. CDI utilizes the formation of electric double layer capacitance (EDLC) at the interfaces of electrode materials. ${ }^{8}$ Once a voltage is applied to the electrodes, ions will follow the electric potential and will be driven to the oppositely charged electrodes, followed by absorption on the electrode surfaces. ${ }^{9}$ After the electrodes are saturated with ions, the electrodes are regenerated by eliminating the electric field. Therefore CDI is also described as an electrosorption technique.

To date, most CDI electrodes consist of carbon materials, such as activated carbon, ${ }^{10}$ carbon aerogel, ${ }^{11,12}$ ordered

\footnotetext{
${ }^{a}$ State Key Laboratory of Chemical Resource Engineering, Beijing University of Chemical Technology, Beijing, 100029, China. E-mail: changzheng@mail.buct.edu. cn; sunxm@mail.buct.edu.cn

${ }^{b}$ Institute of Inorganic Chemistry, RWTH Aachen University, Aachen, 52074, Germany ${ }^{c}$ Maoming Branch R\&D Institute, SINOPEC, Maoming, 525011, China

$\dagger$ Electronic supplementary information (ESI) available. See DOI: 10.1039/c5ta01036e
}

mesoporous carbon, ${ }^{13,14}$ carbon nanotube, ${ }^{15-17}$ graphene,${ }^{18-20}$ and their composites, ${ }^{21-24}$ which take advantage of carbon's high specific surface area (SSA), excellent electronic conductivity, manageable pore structure, and extremely high durability. For the emerging desalination technology, CDI electrodes are still in the developing stage. Recently, the excellent CDI performance of hierarchical porous carbon has drawn a lot of attention. ${ }^{25}$ In addition to increasing SSA and exposing more sites for ions to adsorb, hierarchical pore structure can facilitate ion transport by providing shorter diffusion pathways and smaller resistances: the ion buffering reservoirs formed in macropores can minimize ion diffusion distances into interior pores; ${ }^{26,27}$ the mesopores guarantee not only a large accessible surface area for ion adsorption but also a low resistant pathway for ion transport through the framework. ${ }^{28-30}$

To optimize desalination performance, numerous efforts have been devoted to three-dimensional (3D) carbon materials with multifold pore structures. A 3D carbon sponge based on reduced graphene oxide exhibited a desalination capacity as high as $8.6 \mathrm{mg} \mathrm{g}^{-1},{ }^{31}$ and an electrosorption capacity of $15.2 \mathrm{mg} \mathrm{g}^{-1}$ was also reported based on mesoporous carbon made out of resorcinol. ${ }^{32}$ However, their capacitances and rates for desalination were insufficiently high and left room for upcoming improvement and development. For instance, by introducing metal oxides, 3D carbon composites showed enhanced CDI performance on electrosorption capacity and capacitive retention. ${ }^{33}$ Therefore, the development of high capacitance and low cost of electrode materials is a key factor for a successful and economic commercial application of CDI in the future. 
Ethylenediaminetetraacetic acid (EDTA), one widely available chelating agent in solution chemistry, has been used as a carbon source for novel carbon electrodes. Our team ${ }^{34}$ firstly reported a one-step pyrolysis of EDTA and melamine in the presence of $\mathrm{KOH}$ and $\mathrm{Co}\left(\mathrm{NO}_{3}\right)_{2} \cdot 6 \mathrm{H}_{2} \mathrm{O}$ to synthesize Co-containing porous carbon, which demonstrated excellent oxygen reduction reaction (ORR) activity. Xu et $a l^{35}$ used a direct pyrolysis of the tetrasodium salt of ethylenediaminetetraacetic acid $\left(\mathrm{Na}_{4}\right.$-EDTA) to synthesize supercapacitor electrodes. Bao et $a .^{36}$ prepared a hierarchical architecture that consisted of ultrathin graphene shells that encapsulated CoNi nanoalloy using a hydrothermal reaction of $\mathrm{Co}^{2+}, \mathrm{Ni}^{2+}$ and $\mathrm{Na}_{4}-$ EDTA followed by an annealing treatment, and studied its electrocatalyst application in the hydrogen evolution reaction (HER). In the pyrolysis process of EDTA, $\mathrm{CO}_{2}$ readily generated from carboxyl groups of EDTA expanded the carbon matrix and greatly increased the volume of the reaction system. ${ }^{34}$ The abundant porosity could be further enhanced by $\mathrm{KOH}$ activation. ${ }^{37}$ In situ $\mathrm{N}$-doping derived from $\mathrm{N}$-containing EDTA precursors realized a homogeneous incorporation of nitrogen into the carbon framework, leading to the enhancement of the capacitance through additional Faradaic reactions as well as the improvement of the wettability with electrolytes. ${ }^{35}$ Therefore, the utilization of EDTA precursors helped the formation of a highly porous structure, large specific surface area and improved electrochemical property of EDTA-derived carbon (EDTA-C). Up to now, no one has investigated the CDI performance and electrochemical properties of EDTA-C in a neutral electrolyte.

Moreover, separation of various metal ions in water systems is becoming increasingly urgent in current environmental processes, like water softening or brackish water deionization. A few studies have investigated ion selectivity in CDI. Xu et al. ${ }^{38}$ showed that multivalent ions were preferentially adsorbed onto the charged carbon electrodes, leading to better capacitance performances than monovalent ions. However, Gabelich et al. ${ }^{39}$ reported that monovalent ions with smaller hydrated radii were preferentially removed from solution over multivalent ions with larger radii. Afterwards, Hou et al. ${ }^{\mathbf{4 0}}$ published that activated carbon electrodes presented better selectivity of smaller ions over larger ions, while divalent ions were more preferentially removed than monovalent ions. Moreover, the initial concentration and the applied voltage always play an important role in ion selectivity. It seems that ionic charge and hydrated radius can influence electrosorption selectivity considering similar feed concentrations, but the interplay between the two is not yet clear. So ion selectivity in CDI is still a great challenge.

In this work, we reported the development of a series of hierarchical porous carbon materials (HPCs) formed in a onestep pyrolysis of EDTA with the help of $\mathrm{KOH}$. Hierarchical porosity was achieved by in situ $\mathrm{CO}_{2}$ release and $\mathrm{KOH}$ activation. Very high specific capacitances were found in both $6 \mathrm{~mol} \mathrm{~L}^{-1}$ $\mathrm{KOH}\left(260 \mathrm{~F} \mathrm{~g}^{-1}\right)$ and $1 \mathrm{~mol} \mathrm{~L}^{-1} \mathrm{NaCl}\left(182 \mathrm{~F} \mathrm{~g}^{-1}\right)$ electrolytes, which were attributed to the high SSA, large pore volume, $\mathrm{N}$-doping and appropriate pore structure of the HPCs. This opened up opportunities for CDI applications. The maximum desalination capacity of the optimized HPCs was $34.27 \mathrm{mg} \mathrm{g}^{-1}$ in $40 \mathrm{mg} \mathrm{L}^{-1} \mathrm{NaCl}$ and they displayed $100 \%$ capacity retention for three cycles. In addition, the electrode materials enabled selective adsorption in competitive multi-ionic solutions depending on the ionic charge and hydrated radius. We concluded that the HPCs prepared by this facile and low-cost procedure could potentially pave the way to technical desalination applications.

\section{Experimental}

Ethylenediaminetetraacetic acid (EDTA) and other chemicals were purchased from Beijing Chemical Reagents Company. Commercial activated carbon was purchased from Shantou Xilong Chemical Factory. All the reagents were used without any purification, and deionized (DI) water was used in all experiments.

\subsection{Synthesis of hierarchical porous carbons (HPCs)}

A certain amount of $\mathrm{KOH}(1.0,2.0,4.0$ or $6.0 \mathrm{~g})$ was ground to a fine powder in an agate mortar and then mixed with EDTA $(4.0 \mathrm{~g})$. The mixture was transferred to a ceramic boat and heated to a certain high temperature $\left(500,600,700\right.$ or $\left.800{ }^{\circ} \mathrm{C}\right)$ for $2 \mathrm{~h}$ with a heating rate of $10{ }^{\circ} \mathrm{C} \min ^{-1}$ under an inert atmosphere $\left(\mathrm{N}_{2}\right)$ in a tube furnace. Afterwards, the product was carefully washed with diluted hydrochloric acid, water and ethanol for several cycles, and then finally dried at $80{ }^{\circ} \mathrm{C}$ overnight. According to the weight ratio of $\mathrm{KOH}$ to EDTA, the samples annealed at $600{ }^{\circ} \mathrm{C}$ were denoted as HPC-0.25, HPC-0.5, HPC-1.0, and HPC-1.5.

For control experiments, commercial activated carbon (AC) after pretreatment with nitric acid and water was used to replace EDTA in the same synthesis process. The heating treatment was at $600{ }^{\circ} \mathrm{C}$ for $2 \mathrm{~h}$. The weight ratios of $\mathrm{KOH}$ to $\mathrm{AC}$ were 0 and 0.5 , respectively denoted as $\mathrm{AC}$ and $\mathrm{AC}-\mathrm{KOH}$.

\subsection{Characterization}

The morphologies of the materials were examined using scanning electron microscopy (Zeiss SUPRA 55). Chemical compositions were determined by X-ray photoelectron spectroscopy (Thermo Electron ESCALAB 250). The nitrogen adsorption and desorption isotherms were measured at $77 \mathrm{~K}$ with a Quantachrome Adsorption Instrument (Quantachrome Autosorb-1CVP). Prior to the measurements, the samples were outgassed at $200{ }^{\circ} \mathrm{C}$ in a vacuum for $3 \mathrm{~h}$. Specific surface area was calculated from the adsorption branch according to the BrunauerEmmett-Teller (BET) method. The pore size distribution plot was derived from the desorption branch of the isotherm based on the Barrett-Joyner-Halenda (BJH) method. The cationic concentrations in multi-ionic solutions were measured by an inductively coupled plasma-atomic emission spectrometer (Thermo, ICAP-6300). Raman spectra were recorded from 100 to $4500 \mathrm{~cm}^{-1}$ on the LabRAM ARAMIS Raman system with a $532 \mathrm{~nm}$ argon ion laser for excitation.

\subsection{Electrode preparation}

The HPCs and ACs were used as electrode materials. In a typical procedure, the HPCs (or ACs), carbon black, and 
polytetrafluoroethylene (PTFE, Aldrich; $60 \mathrm{wt} \%$ dispersion in water) were mixed ( $80 \mathrm{wt} \%, 15 \mathrm{wt} \%$ and $5 \mathrm{wt} \%$, respectively) in an appropriate volume of ethanol and then sonicated for at least $1 \mathrm{~h}$. The slurry containing the solid electrode materials was pressed on a graphite sheet. The fabricated electrodes were dried at $80{ }^{\circ} \mathrm{C}$ overnight to remove any remaining solvent. The small electrodes (with a mass of $5 \mathrm{mg}$ and a size of $1 \mathrm{~cm} \times 1 \mathrm{~cm}$ $\times 0.2 \mathrm{~mm}$ ) and large electrodes (with a mass of $100 \mathrm{mg}$ and a size of $6 \mathrm{~cm} \times 7 \mathrm{~cm} \times 0.2 \mathrm{~mm}$ ) were used for electrochemical measurements and capacitive desalination experiments, respectively.

\subsection{Electrochemical measurements}

The capacitance examination (CV), galvanostatic chargedischarge (GCD) and electrochemical impedance spectroscopy (EIS) measurements were performed in $1.0 \mathrm{~mol} \mathrm{~L}^{-1} \mathrm{NaCl}$ aqueous solutions on a $\mathrm{CHI}$ 660D electrochemical station (Chenhua Instrument Company), using a three-electrode configuration that consisted of a working electrode, a graphite electrode, and a calomel reference electrode. CV and GCD measurements were carried out between 0 and $1.0 \mathrm{~V}$, and the frequency range of EIS impedance was $100 \mathrm{kHz}-0.01 \mathrm{~Hz}$. The cycling ability was measured at a current density of $0.5 \mathrm{~A} \mathrm{~g}^{-1}$ in a potential window of $0-1.0 \mathrm{~V}$. The specific capacitances $(C)$ were calculated from GCD curves according to the equation:

$$
C=I \Delta t / m \Delta V
$$

where $I$ is the discharge current density, $\Delta t$ is the discharge time, $m$ is the mass of the active materials, and $\Delta V$ is the discharge voltage difference.

\subsection{Capacitive desalination experiments}

In a typical CDI measurement, $100 \mathrm{~mL}$ of $\mathrm{NaCl}$ aqueous solution with an initial conductivity of $90 \mu \mathrm{S} \mathrm{cm}^{-1}\left(40 \mathrm{mg} \mathrm{L}^{-1}\right)$ in a beaker was continuously fed into the CDI unit using a peristaltic pump with a constant flow rate of $25 \mathrm{~mL} \mathrm{~min}^{-1}$ and the effluent was returned to this beaker. Meanwhile, the applied voltage on the CDI unit was adjusted from 1.2 to 1.4 to $1.6 \mathrm{~V}$. The initial concentration of $\mathrm{NaCl}$ aqueous solution was changed to 500 , 1000 or $5850 \mathrm{mg} \mathrm{L}^{-1}$. The solution concentration was monitored by a conductivity meter (Type 308F, Leici Company) located at the outlet of the CDI unit. The desalination capacities $(W)$ were calculated according to the equation:

$$
W=\left(C_{0}-C\right) V / m
$$

where $C_{0}$ and $C$ are the initial and final concentrations, respectively, calculated from the corresponding conductivities, $V$ is the total solution volume, and $m$ is the total mass of the electrode materials. The accumulated ion removal rate was given by the accumulated mass of deionized $\mathrm{NaCl}(\mathrm{mg})$ per total electrode material mass $(\mathrm{g})$ divided by the operation time (s). ${ }^{41}$ In the selectivity measurements, $100 \mathrm{~mL}$ of multi-ionic solutions were used containing $\mathrm{LiCl}, \mathrm{KCl}, \mathrm{RbCl}, \mathrm{MgCl}_{2}$ or $\mathrm{AlCl}_{3}$, in which every cationic concentration was $0.45 \mathrm{mmol} \mathrm{L}^{-1}$.

\section{Results and discussion}

The resulting HPCs materials were verified to have hierarchical pore structures, as shown in Fig. 1. The HPC-0.25, HPC-0.5, HPC-1.0, and HPC-1.5 samples were prepared under the same conditions except for the different weight ratio of $\mathrm{KOH}$ to EDTA. Scanning electron microscopy (SEM) images clearly exhibited macroporous structures with well-defined interconnected pores at a size range from 5 to $10 \mu \mathrm{m}$ (Fig. 1a-d). In high magnification SEM images (Fig. S1a-d, ESI $\dagger$ ), more pores could be found on the walls building up the macropores. The porous structures of the HPCs were further evaluated by $\mathrm{N}_{2}$ adsorption-desorption measurements, and the results are shown in Fig. 1e and $\mathrm{f}$ and Table 1. According to IUPAC classification, the strip hysteresis loops in Fig. 1e indicated that the HPCs mainly consisted of mesopores. Meanwhile, the isotherms presented certain volume adsorption when the $P / P_{0}$ decreased to 0.4 and below, suggesting the microporous channel distribution. Thus, the HPCs possessed a hierarchical porous network in terms of micro-, meso- and macro-pores as well as an abundant porosity, which would benefit ion adsorption, diffusion and transport. ${ }^{\mathbf{4 2}}$ The weight ratio of $\mathrm{KOH}$ to EDTA had an obvious effect on the SSA and pore structure. The HPC-0.25, HPC-0.5, HPC-1.0, and HPC-1.5 gave SSAs of 1419.96, 2185.71, 1599.59, and $871.44 \mathrm{~m}^{2}$ $\mathrm{g}^{-1}$, respectively. Their mesopore sizes were lying in a range from 3.0 to $4.0 \mathrm{~nm}$ (Fig. 1f), while their specific pore volumes were significantly different. For instance, the pore volume of the HPC-0.5 (1.368 $\left.\mathrm{cm}^{3} \mathrm{~g}^{-1}\right)$ was much larger than that of the HPC-1.5 $\left(0.557 \mathrm{~cm}^{3} \mathrm{~g}^{-1}\right)$ despite their similarity in average pore size (about $3.7 \mathrm{~nm}$ ), revealing that their difference in pore structure was probably relevant to the $\mathrm{KOH}$ effect on micropores. Generally, large SSA and appropriate pore size are crucial for electrode materials ${ }^{43}$ so that the HPCs are expected to have a superior capacitance. ${ }^{21}$ Moreover, the graphitization degrees of the HPCs were investigated using Raman (Fig. S2 $\dagger$ ). The lowest intensity ratio of $\mathrm{D}$ band to $\mathrm{G}$ band $\left(I_{\mathrm{D}} / I_{\mathrm{G}}\right)$ for the HPC-0.5 confirmed it to be the most highly graphitized, which was normally treated as a sign of enhanced electrical conductivity. ${ }^{\mathbf{4 4}}$ The element contents calculated from XPS data are listed in Table 1. The C1s spectra of the HPCs are illustrated in Fig. S3a $\dagger$ and split to four peaks, respectively attributed to $\mathrm{sp}^{2}$ carbon

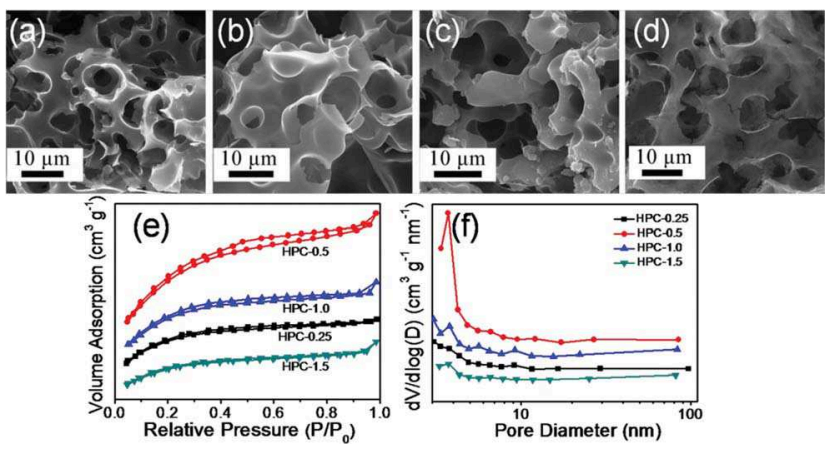

Fig. 1 SEM images of (a) HPC-0.25, (b) HPC-0.5, (c) HPC-1.0 and (d) HPC-1.5. (e) $\mathrm{N}_{2}$ adsorption-desorption isotherms and (f) pore size distributions of the HPCs. 
Table 1 Structural and constituent data of HPC-0.25, HPC-0.5, HPC-1.0 and HPC-1.5

\begin{tabular}{lllllrr}
\hline HPC- & $S_{\text {BET }} \mathrm{m}^{2} \mathrm{~g}^{-1}$ & $D_{\text {me }} \mathrm{nm}$ & $V_{\text {total }} \mathrm{cm}^{3} \mathrm{~g}^{-1}$ & C content\% & N content\% & O content\% \\
\hline 0.25 & 1419.96 & 3.06 & 0.777 & 85.00 & 4.16 & 10.84 \\
0.5 & 2185.71 & 3.73 & 1.368 & 85.89 & 8.74 & 10.37 \\
1.0 & 1599.59 & 3.05 & 0.936 & 86.31 & 6.45 & 9.07 \\
1.5 & 871.44 & 3.74 & 0.557 & & 8.90
\end{tabular}

(284.9 eV), carbon in $\mathrm{C}-\mathrm{N}(286.5 \mathrm{eV})$, carbon in carbonyl (287.7 eV), and carbon in carboxyl or ester group (289.6 eV). Less $\mathrm{C}-\mathrm{O}$ groups were observed with more $\mathrm{KOH}$ being added, suggesting a higher carbonization/defunctionalization degree during $\mathrm{KOH}$ activation at high temperature. The HPC-0.5 showed a relatively high $\mathrm{O} / \mathrm{C}$ ratio, which meant a high level of functionalization. The combination of good conductivity and reasonable surface functionalization would yield the best capacitive performance of the HPC-0.5. The N1s spectra (Fig. S3b $\dagger$ ) could be fitted with three signals of pyridine $\mathrm{N}$, pyrrolic $\mathrm{N}$ and quaternary- $\mathrm{N}$ at $\sim 398.6,400.1$ and $401.1 \mathrm{eV}$, respectively. ${ }^{45-47}$ The $\mathrm{N}$ presence suggested that $\mathrm{N}$ atoms source from EDTA precursors had been incorporated into the carbon framework, which would benefit the capacitive performance of the HPCs. ${ }^{\mathbf{4 8 , 4 9}}$ Therefore, the hierarchical porous structure, high specific surface area, appropriate pore size, reasonable graphitization degree and in situ N-doping of the HPCs made them ideal as electrode materials for supercapacitor and CDI processes.

The supercapacitor performance of the HPCs was initially evaluated in $1 \mathrm{~mol} \mathrm{~L}^{-1} \mathrm{NaCl}$ electrolyte considering that the capacitive behavior in $\mathrm{NaCl}$ aqueous solution was closely related to the CDI performance mainly based on an EDLC principle. Some experimental conditions were optimized depending on the electrochemical property. Firstly, the effect of the annealing temperature of the system was investigated. Cyclic voltammetry (CV) measurements with a scan rate of $50 \mathrm{mV} \mathrm{s}^{-1}$ for the HPC-0.5 samples annealed at 500, 600 and $700{ }^{\circ} \mathrm{C}$ are depicted in Fig. 2a. The HPC-0.5 annealed at $600{ }^{\circ} \mathrm{C}$ displayed the largest voltammogram area, which was simply translated into the highest capacitance. The galvanostatic charge-discharge (GCD) curves at a current density of $0.5 \mathrm{~A} \mathrm{~g}^{-1}$ (Fig. 2b) also confirmed the optimal annealing temperature. When the annealing temperature decreased to $500{ }^{\circ} \mathrm{C}$, the capacitance reduced sharply, which was attributed to the poor conductivity and low porosity of the electrode material after an inadequate heating treatment. However, the high annealing temperature of $700{ }^{\circ} \mathrm{C}$ could significantly increase the $\mathrm{C}-\mathrm{KOH}$ reaction rate and then cause the burn-off of some carbon structures and the widening of micropores, which led to the decrease of useful pores for the capacitance. ${ }^{50}$ The capacitance evolution in alkaline electrolyte also agreed with it very well, as shown in Fig. S4a. $\dagger$ Therefore, we identified $600{ }^{\circ} \mathrm{C}$ as the optimal annealing temperature for experiments afterwards in this work. Subsequently, the CV measurements of the HPCs with different weight ratios of $\mathrm{KOH}$ to EDTA were carried out at a scanning rate of $100 \mathrm{mV} \mathrm{s}^{-1}$ and recorded in Fig. 2c. Their GCD curves at a current density of $0.5 \mathrm{~A} \mathrm{~g}^{-1}$ were shown in Fig. 2d. The HPC-0.5 displayed the largest voltammogram area and the longest charge-discharge time, suggesting the highest capacitance. Revealed by the GCD curves, HPC-0.25, HPC-0.5, HPC-1.0 and HPC-1.5 had specific capacitances of $101.8,182.2,156.9$ and $70.94 \mathrm{~F} \mathrm{~g}^{-1}$ in $1 \mathrm{~mol} \mathrm{~L}^{-1}$ $\mathrm{NaCl}$, respectively. The small deviation from the linear GCD curves suggested that the capacitance mainly resulted from EDLC, partly combining with some contribution of the heteroatoms (doped $\mathrm{N}$ ) in electrode materials. It is well known that nitrogen doping is a simple but effective process to improve the electrochemical performance of carbon materials. ${ }^{51}$ As shown in Fig. 2e, all of the iR drop curves of the HPCs almost linearly increased with the current density increasing and the HPC-0.5 showed the smallest iR drop by comparison with the other analogues, suggesting a facilitated electronic/ionic transfer and low contact resistance. ${ }^{43}$ In the electrochemical impedance spectroscopy (EIS) of the HPCs (Fig. S5†), HPC-0.5 presented the smallest semicircle of the Nyquist plot in the high-frequency region, which further proved the lowest charge transfer resistance. ${ }^{52}$ Considering both capacitance functionality and inner
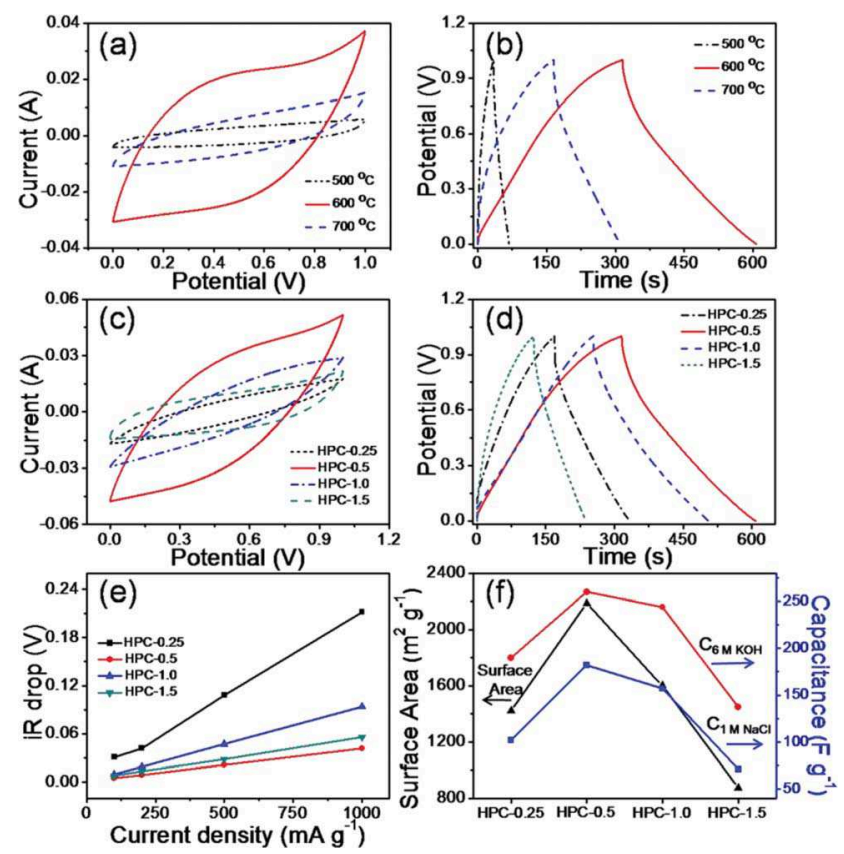

Fig. 2 Electrochemical performances of the HPCs in $1 \mathrm{~mol} \mathrm{~L}^{-1} \mathrm{NaCl}$ (a) CV measurements (a scan rate of $50 \mathrm{mV} \mathrm{s}^{-1}$ ) and (b) GCD curves (a current density of $0.5 \mathrm{~A} \mathrm{~g}^{-1}$ ) of the HPC-0.5 samples annealed at different temperatures. (c) CV measurements (a scan rate of $100 \mathrm{mV} \mathrm{s}^{-1}$ ), (d) GCD curves (a current density of $0.5 \mathrm{~A} \mathrm{~g}^{-1}$ ), (e) iR drop curves, and (f) the specific capacitance and SSA evolution of HPC-0.25, HPC-0.5, HPC-1.0 and HPC-1.5. 
resistance, the optimal weight ratio of $\mathrm{KOH}$ to EDTA was identified as $1: 2$. The evolution of specific capacitances and SSAs of the HPCs is reflected in Fig. 2f. It was observed that the specific capacitances in alkaline or neutral electrolyte displayed the same trend with the SSAs: a gradual decrease from HPC-0.5, HPC-1.0, HPC-0.25 to HPC-1.5, which indicated that the SSA influenced electrochemical property as a crucial structural parameter because the large exposed area could provide sufficient sites for ions absorption in EDLC. ${ }^{43}$

To obtain further insights into the optimal HPC-0.5, its electrochemical property was carefully investigated in $1 \mathrm{~mol} \mathrm{~L}^{-1}$ $\mathrm{NaCl}$ solution. Fig. 3a depicts the CV curves of the HPC-0.5 at different scan rates from 1 to $100 \mathrm{mV} \mathrm{s}^{-1}$. The curves maintained quasi-rectangular shaped voltammetry characteristics at low scan rates $\left(<50 \mathrm{mV} \mathrm{s}^{-1}\right)$, indicating the EDLC behavior of the carbon composites. However, when the scan rate increased to $50 \mathrm{mV} \mathrm{s}^{-1}$ and above, the shapes exhibited increased distortion from the typical rectangular shape, which mainly resulted from the inherent inner resistance as well as the pseudocapacitance contribution related to the heteroatoms (doped N). ${ }^{34,35,53,54}$ For comparison with conventional carbon electrodes, activated carbon (AC) electrodes before and after $\mathrm{KOH}$ activation were evaluated as control samples in the same system. Estimated from GCD measurements, the specific capacitances of the HPC-0.5, AC and AC-KOH electrodes at different current densities are displayed in Fig. 3b. At $0.5 \mathrm{~A} \mathrm{~g}^{-1}$, the HPC-0.5 had a high specific capacitance of $182.2 \mathrm{~F} \mathrm{~g}^{-1}$, which surpassed $68.5 \mathrm{~F} \mathrm{~g}^{-1}$ of $\mathrm{AC}, 86.0 \mathrm{~F} \mathrm{~g}^{-1}$ of $\mathrm{AC}-\mathrm{KOH}$ and most of the other currently reported data of CDI electrode materials. ${ }^{30}$ It was consistent with the lower specific surface areas and less pore structures of $\mathrm{AC}$ and $\mathrm{AC}-\mathrm{KOH}$, as shown in Table S1 and Fig. S6. $\uparrow$ Moreover, at a higher current density of $2.0 \mathrm{~A} \mathrm{~g}^{-1}$, the specific capacitances of the HPC-0.5, AC and AC- $\mathrm{KOH}$ electrodes were 109.5, 38.0, and $52.0 \mathrm{~F} \mathrm{~g}^{-1}$, respectively, which corresponded to the capacitance retentions of $60.1 \%, 55.5 \%$, and $60.5 \%$. HPC- 0.5 showed similar capacitance retention with that of $\mathrm{AC}$ and $\mathrm{AC}-\mathrm{KOH}$
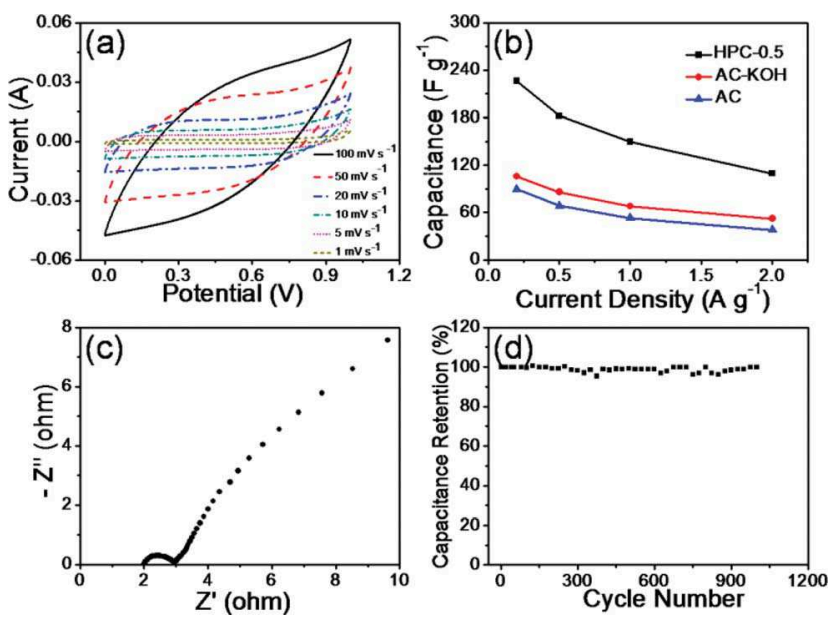

Fig. 3 Electrochemical performances of HPC- 0.5 in $1 \mathrm{~mol} \mathrm{~L}^{-1} \mathrm{NaCl}$ (a) CV curves at different scan rates, (b) specific capacitances of HPC-0.5, $\mathrm{AC}$ and $\mathrm{AC}-\mathrm{KOH}$ at different current densities, (c) EIS measurement, and (d) cycling stability at a current density of $0.5 \mathrm{~A} \mathrm{~g}^{-1}$. possessing pure EDLC, implying a quick charge propagation capability of both double layer capacitance and pseudocapacitance, and a good rate capability. ${ }^{55-57}$ In the EIS curve (Fig. 3c), a small semicircle of the Nyquist plot was observed in the highfrequency region, which confirmed a relatively low contact resistance of HPC-0.5 as one of the explanations for the high capacitance. ${ }^{58}$ HPC-0.5 also showed a remarkable cycling stability, exhibiting a 96\% capacitance retention when cycling 1000 times at $0.5 \mathrm{~A} \mathrm{~g}^{-1}$ (Fig. 3d). Considering these remarkable electrochemical properties of the HPCs in neutral solution, we could expect much better supercapacitor performances in alkaline electrolyte. ${ }^{59-61}$ For instance, HPC-0.5 had a very high specific capacitance of $260 \mathrm{~F} \mathrm{~g}^{-1}$ at $0.5 \mathrm{~A} \mathrm{~g}^{-1}$ and $235 \mathrm{~F} \mathrm{~g}^{-1}$ at $1 \mathrm{~A} \mathrm{~g}^{-1}$ in $6.0 \mathrm{~mol} \mathrm{~L}^{-1} \mathrm{KOH}$ (for details, please refer to Fig. $\mathrm{S} 4 \dagger$ and corresponding description). Therefore, the HPCs with hierarchical pore structures, large SSAs and good capacitive performances would be desirable for a high-efficiency and longterm operation of CDI. ${ }^{\mathbf{2}}$

The desalination performances of the HPCs were evaluated in $\mathrm{NaCl}$ aqueous solutions using a bench-scale CDI testing apparatus, which consisted of a pump, a conductivity meter, an electrical power supply, a self-made CDI unit and a feed water reservoir (as shown in Fig. 4). The change in conductivity was closely related to the solution concentration, which helped to monitor ionic adsorption-desorption processes of the electrode materials. The impact of applied voltage on CDI performance was firstly investigated in $26 \mathrm{mg} \mathrm{L}^{-1} \mathrm{NaCl}$ solution using the HPC-0.5 electrode at $1.2 \mathrm{~V}, 1.4 \mathrm{~V}$ and $1.6 \mathrm{~V}$ (Fig. 5a). To study physical adsorption behavior, the voltage was not applied in the first 20 min while the pump worked. It was exciting to find that the conductivity of every system hardly decreased at this stage, suggesting that the physical adsorption was almost ignored. Generally, relatively obvious physical adsorption always limits the applications of carbon materials. ${ }^{63}$ By comparison with these common carbon materials, the low physical adsorption capacity of the HPCs would benefit the enhancement of electrosorption capacity and recycling stability. Once the voltage

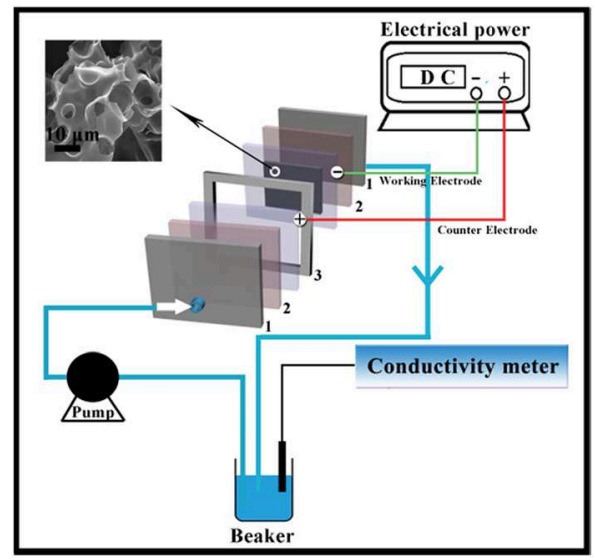

Fig. 4 Schematic of the CDI testing apparatus: electrical power supply, conductivity meter, feed water reservoir, pump, and the CDI unit (1-plastic plate; 2 -silicon rubber plate; 3 -plastic spacer; working electrode; counter electrode). 

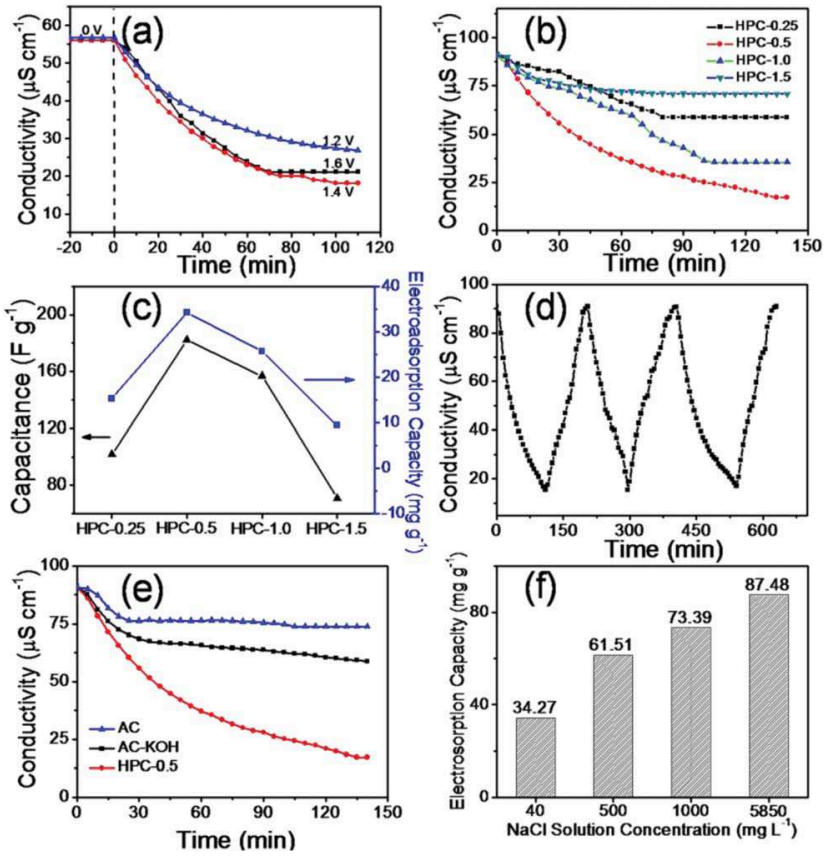

Fig. $5 \mathrm{CDI}$ performances of the HPCs electrodes in $40 \mathrm{mg} \mathrm{L}^{-1} \mathrm{NaCl}$ at $1.4 \mathrm{~V}$ (a) the conductivity transients of HPC- 0.5 at different applied voltages ( $26 \mathrm{mg} \mathrm{L}^{-1} \mathrm{NaCl}$ ), (b) the conductivity transients of HPC- 0.25 , HPC-0.5, HPC-1.0 and HPC-1.5, (c) the specific capacitance and desalination capacity evolution of HPC-0.25, HPC-0.5, HPC-1.0 and HPC-1.5, (d) the cycling stability of HPC-0.5, (e) the conductivity transients of $\mathrm{HPC}-0.5, \mathrm{AC}$ and $\mathrm{AC}-\mathrm{KOH}$, and ( $\mathrm{f}$ ) the desalination capacities of HPC-0.5 in different concentrations of $\mathrm{NaCl}$.

was applied, the conductivity of every system decreased sharply and then reached a plateau within 100 min with the electrode being saturated. The applied voltage of $1.4 \mathrm{~V}$ would be used in the following experiments rather than $1.2 \mathrm{~V}$ and $1.6 \mathrm{~V}$, because it induced sufficiently high electrostatic force but did not split water into $\mathrm{O}_{2}$ and $\mathrm{H}_{2} \cdot{ }^{64}$ Subsequently, the CDI performances over different HPCs electrodes were measured in $40 \mathrm{mg} \mathrm{L}^{-1}$ $\mathrm{NaCl}$ solution. As shown in Fig. 5b, they all presented great CDI performances. After $120 \mathrm{~min}$, the desalination capacities (also known as electrosorption capacity) of the HPC-0.25, HPC-0.5, HPC-1.0 and HPC-1.5 electrodes were 15.35, 34.27, 25.68, and $9.41 \mathrm{mg} \mathrm{g}^{-1}$, respectively. As we expected, the HPC-0.5 electrode performed much better than any of the others, and the desalination capacity of $34.27 \mathrm{mg} \mathrm{g}^{-1}$ exceeded most of the reports about CDI electrode materials, as listed in Table $2 .^{31,33,65-68}$ The conductivity of the system decreased sharply in the first $80 \mathrm{~min}$, indicating that the desalination process proceeded relatively quickly. Finally, the conductivity reduced from 90 to $17 \mu \mathrm{S} \mathrm{cm}{ }^{-1}$ and thus the removal efficiency was about $80 \%$. To reveal the "intrinsic factors" that dominate the excellent CDI performances of the HPCs, the desalination capacity and specific capacitance evolutions of HPC-0.25, HPC-0.5, HPC-1.0 and HPC-1.5 are simultaneously shown in Fig. 5c. HPC-0.5 displayed the peak maximum in both of the curves, in good agreement with the change in structural characters such as SSA and pore volume. Thus, the high specific capacitance, large SSA, and appropriate pore structure were considered as the main factors responsible for the superior CDI capacity of the HPCs.

As another important criterion, the cycling stability of the HPC-0.5 is measured and the conductivity transients over three consecutive adsorption-desorption cycles are presented in Fig. 5d. The desorption process was simply achieved by removing the applied voltage. The conductivity was perfectly reproducible and the conductivity retention was above $98 \%$ after every adsorption-desorption cycle, indicating a remarkable stability of the HPC-0.5 electrode. The complete release of these adsorbed ions might be attributed to the very low physical adsorption capacity of HPC- 0.5 and the CDI capacity could be regenerated very well without any driving energy and secondary pollution, which is important for a large-scaled application. The CDI performance comparison of the HPC-0.5 and conventional AC electrodes was carried out and is shown in Fig. 5e. The desalination capacities of $\mathrm{AC}$ and $\mathrm{AC}-\mathrm{KOH}$ were 8.16 and $15.79 \mathrm{mg} \mathrm{g}^{-1}$, respectively, less than $34.27 \mathrm{mg} \mathrm{g}^{-1}$ of HPC-0.5. Fig. S7 $\uparrow$ shows their time-dependent accumulated ion removal rates. The maximum ion removal rates of HPC-0.5, AC and $\mathrm{AC}-\mathrm{KOH}$, calculated from the peaks of curves ${ }^{41}$ were 0.010 , 0.005 and $0.008 \mathrm{mg} \mathrm{g}^{-1} \mathrm{~s}^{-1}$, respectively. The CDI performances of the HPCs surpassed those of conventional AC materials. Moreover, the study in high-concentration salt solutions is significant for the development of the CDI technique. ${ }^{41}$ As shown in Fig. S8, $\uparrow$ the HPC-0.5 electrode successfully completed desalination processes within $120 \mathrm{~min}$ in 500, 1000 and $5850 \mathrm{mg} \mathrm{L}^{-1} \mathrm{NaCl}$ solutions and the corresponding desalination capacities were $61.51,73.39$ and $87.48 \mathrm{mg} \mathrm{g}^{-1}$, respectively (Fig. 5f). The maximum ion removal rates also increased with increasing concentration, for instance, $0.035 \mathrm{mg} \mathrm{g}^{-1} \mathrm{~s}^{-1}$ in $500 \mathrm{mg} \mathrm{L}^{-1}$, and $0.064 \mathrm{mg} \mathrm{g}^{-1} \mathrm{~s}^{-1}$ in $5850 \mathrm{mg} \mathrm{L}^{-1} \mathrm{NaCl}$. The results indicated that the HPCs materials were efficient in

Table 2 Electrosorption data in our work and some references

\begin{tabular}{|c|c|c|c|c|}
\hline Sample & Applied voltage (V) & Initial salt concentration & Desalination capacity $\left(\mathrm{mg} \mathrm{g}^{-1}\right)$ & Ref. \\
\hline PPy/PANI & 1.4 & $500 \mathrm{mg} \mathrm{L}^{-1}$ & 197.8 & 65 \\
\hline $\mathrm{GA} / \mathrm{TiO}_{2}$ & 1.2 & $500 \mathrm{mg} \mathrm{L}^{-1}$ & 24.2 & 33 \\
\hline $\mathrm{OMC} / \mathrm{CNT}$ & 1.2 & $40 \mathrm{mg} \mathrm{L}^{-1}$ & 0.686 & 66 \\
\hline $\mathrm{Gr} / \mathrm{SnO}_{2}$ & 1.4 & $20-30 \mathrm{mg} \mathrm{L}^{-1}\left(61 \mu \mathrm{S} \mathrm{cm}^{-1}\right)$ & 1.49 & 67 \\
\hline GNFs & 2.0 & $25 \mathrm{mg} \mathrm{L}^{-1}$ & 1.21 & 68 \\
\hline
\end{tabular}



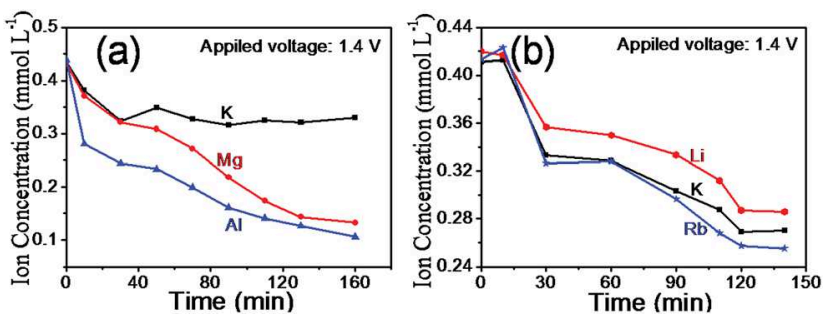

Fig. 6 Ion concentration evolution in multi-ionic solutions: (a) $\mathrm{Al}^{3+}$, $\mathrm{Mg}^{2+}, \mathrm{K}^{+}$, and (b) $\mathrm{Li}^{+}, \mathrm{K}^{+}, \mathrm{Rb}^{+}$.

treating high-concentration salt solutions. In summary, the HPCs would hopefully become a kind of potential CDI electrode material because of their superior performances, including reduced physical adsorption, high desalination capacity, and facile regeneration.

To investigate the ion selectivity of the HPCs, some typical cations were selected including $\mathrm{Li}^{+}, \mathrm{K}^{+}, \mathrm{Rb}^{+}, \mathrm{Mg}^{2+}$ and $\mathrm{Al}^{3+}$. Firstly, $\mathrm{K}^{+}, \mathrm{Mg}^{2+}$ and $\mathrm{Al}^{3+}$ with different ionic charges were studied in a mixed solution, in which every ionic concentration was equal to $0.45 \mathrm{mmol} \mathrm{L}^{-1}$ and $r_{\left(\mathrm{Al}^{3+} \text {, hydrated, } 0.475 \mathrm{~nm}\right)}>r_{\left(\mathrm{Mg}^{2+} \text {, }\right.}$

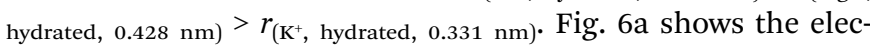
trosorption kinetic curves of these coexisting cations using the HPC-0.5 electrode at an applied voltage of $1.4 \mathrm{~V}$. By comparison with multivalent ions, $\mathrm{K}^{+}$presented the smallest capacity and lowest rate, suggesting that ionic charge could dramatically influence an electrosorption process. Moreover, $\mathrm{Al}^{3+}$ displayed a higher removal rate than $\mathrm{Mg}^{2+}$ though their difference in electrosorption capacity was not significant. The fast removal of $\mathrm{Al}^{3+}$ was probably related to the higher ratio of its surface charge to hydrated radius (as shown in Table $52 \dagger$ ), which could increase the charge density and thus enhance the electrostatic interaction between the $\mathrm{Al}^{3+}$ ion and the oppositely charged electrode. However, after some time, small-sized $\mathrm{Mg}^{2+}$ ions could finally occupy some extra small pores not being utilized by large-sized $\mathrm{Al}^{3+}$ ions, leading to the different electrosorption capacities of $\mathrm{Al}^{3+}$ and $\mathrm{Mg}^{2+}$. The effects of ionic charge and hydrated radius were further confirmed in Fig. 6b, in which the electrosorption performances of coexisting $\mathrm{Li}^{+}, \mathrm{K}^{+}$and $\mathrm{Rb}^{+}$belonging to the same congener were displayed. Three monovalent ions have different hydrated radii: $r_{\left(\mathrm{Li}^{+} \text {, hydrated, } 0.382 \mathrm{~nm}\right)}>r_{\left(\mathrm{K}^{+} \text {, hydrated, } 0.331\right.}$ $\mathrm{nm})>r_{\left(\mathrm{Rb}^{+} \text {, hydrated, } 0.329 \mathrm{~nm}\right)}$. The electrosorption rate and capacity gradually increased with decreasing hydrated radius of three monovalent ions, which agreed perfectly with the above result. The study on ion selectivity indicated that the HPCs could successfully achieve the separation of ions by kinetic behavior difference, or static capacitance difference, which would be meaningful for water desalination and softening, and metal source reuse.

\section{Conclusions}

The hierarchical porous carbon materials (HPCs) were synthesized in the one-step thermal treatment of EDTA with the help of $\mathrm{KOH}$ and firstly applied as CDI electrodes. Due to the highly porous structure formed by gas emission and $\mathrm{KOH}$ activation, the HPCs displayed large specific surface areas and high specific capacitances. The optimized HPC- 0.5 synthesized at $600{ }^{\circ} \mathrm{C}$ with a $\mathrm{KOH} /$ EDTA weight ratio of 0.5 exhibited superior specific capacities in both $6 \mathrm{~mol} \mathrm{~L}{ }^{-1} \mathrm{KOH}\left(260 \mathrm{~F} \mathrm{~g}^{-1}\right)$ and $1 \mathrm{~mol} \mathrm{~L}^{-1}$ $\mathrm{NaCl}\left(182 \mathrm{~F} \mathrm{~g}^{-1}\right)$. Furthermore, it demonstrated an impressive desalination capacity of $34.27 \mathrm{mg} \mathrm{g}^{-1}$ in $40 \mathrm{mg} \mathrm{L}^{-1} \mathrm{NaCl}$ solution. The physical adsorption of ions could almost be ignored and the cycling stability was comparatively high. Perfect desalination performance coupled with cost-effective preparation procedure and reasonable regeneration suggested a great potential application of the HPCs as practical CDI electrode materials. The research also displayed the strong correlation between desalination capacity and physicochemical property of the HPCs. Finally, the HPCs presented ion selectivity by electrosorption performance comparison of coexisting $\mathrm{Li}^{+}, \mathrm{K}^{+}, \mathrm{Rb}^{+}$, $\mathrm{Mg}^{2+}$ or $\mathrm{Al}^{3+}$ ions depending on the combined effect of ionic charge and hydrated radius.

\section{Acknowledgements}

This work was financially supported by the National Natural Science Foundation of China (NSFC), the 973 Program (2011 CBA00503, 2011 CB932403), Changjiang Scholars and Innovative Research Team in University (IRT1205), the China Scholarship Council (CSC), the Fundamental Research Funds for the Central Universities (YS1406).

\section{Notes and references}

1 M. A. Shannon, P. W. Bohn, M. Elimelech, J. G. Georgiadis, B. J. Marinas and A. M. Mayes, Nature, 2008, 452, 301-310.

2 N. Savage and M. S. Diallo, J. Nanopart. Res., 2005, 7, 331342.

3 Y. Oren, Desalination, 2008, 228, 10-29.

4 X. Lei, B. Wang, J. Liu, Z. Ye, Z. Chang, M. Jiang and X. Sun, RSC Adv., 2014, 4, 41642-41648.

5 H. L. Yang, C. Huang and J. C.-T. Lin, Desalination, 2010, 250, 548-552.

6 M. Turek, Desalination, 2002, 153, 377-381.

7 M. H. Eikani, F. Golmohammad, S. Rowshanzamir and M. Mirza, Flavour Fragrance J., 2005, 20, 555-558.

8 R. Zhao, P. M. Biesheuvel, H. Miedema, H. Bruning and A. van der Wal, J. Phys. Chem. Lett., 2010, 1, 205-210.

9 H. H. Jung, S. W. Hwang, S. H. Hyun, K.-H. Lee and G.-T. Kim, Desalination, 2007, 216, 377-385.

10 I. Villar, S. Roldan, V. Ruiz, M. Granda, C. Blanco, R. Menéndez and R. Santamaría, Energy Fuels, 2010, 24, 3329-3333.

11 J. C. Farmer, D. V. Fix, G. V. Mack, R. W. Pekala and J. F. Poco, J. Electrochem. Soc., 1996, 143, 159-169.

12 M. Zafra, P. Lavela, G. Rasines, C. Macías, J. Tirado and C. Ania, Electrochim. Acta, 2014, 135, 208-216.

13 L. Zou, L. Li, H. Song and G. Morris, Water Res., 2008, 42, 2340-2348.

14 Z. Peng, D. Zhang, L. Shi, T. Yan, S. Yuan, H. Li, R. Gao and J. Fang, J. Phys. Chem. C, 2011, 115, 17068-17076. 
15 Y. Liu, W. Ma, Z. Cheng, J. Xu, R. Wang and X. Gang, Desalination, 2013, 326, 109-114.

16 L. Fan, C. Feng, W. Zhao, L. Qian, Y. Wang and Y. Li, Nano Lett., 2012, 12, 3668-3673.

17 K. Shi, M. Ren and I. Zhitomirsky, ACS Sustainable Chem. Eng., 2014, 2, 1289-1298.

18 Z. Wang, B. Dou, L. Zheng, G. Zhang, Z. Liu and Z. Hao, Desalination, 2012, 299, 96-102.

19 H. Wang, D. Zhang, T. Yan, X. Wen, J. Zhang, L. Shi and Q. Zhong, J. Mater. Chem. A, 2013, 1, 11778-11789.

20 B. Jia and L. Zou, Sci. Adv. Mater., 2013, 5, 1111-1116.

21 X. Wen, D. Zhang, T. Yan, J. Zhang and L. Shi, J. Mater. Chem. A, 2013, 1, 12334-12344.

22 C. Yan, L. Zou and R. Short, Desalination, 2012, 290, 125-129.

23 H. Wang, L. Shi, T. Yan, J. Zhang, Q. Zhong and D. Zhang, J. Mater. Chem. A, 2014, 2, 4739-4750.

24 D. Zhang, T. Yan, L. Shi, Z. Peng, X. Wen and J. Zhang, J. Mater. Chem., 2012, 22, 14696-14704.

25 S. J. Yang, T. Kim, K. Lee, Y. S. Kim, J. Yoon and C. R. Park, Carbon, 2014, 71, 294-302.

26 R. A. Rica, D. Brogioli, R. Ziano, D. Salerno and F. Mantegazza, J. Phys. Chem. C, 2012, 116, 16934-16938.

27 Z. Wang, D. Xu, H. Wang, Z. Wu and X. Zhang, ACS Nano, 2013, 7, 2422-2430.

28 Y. Li, Z. Li and P. K. Shen, Adv. Mater., 2013, 25, 2474-2480. 29 D. W. Wang, F. Li, M. Liu, G. Q. Lu and H. M. Cheng, Angew. Chem., Int. Ed. Engl., 2008, 47, 373-376.

30 D. Zhang, X. Wen, L. Shi, T. Yan and J. Zhang, Nanoscale, 2012, 4, 5440-5446.

31 B. Jia and L. Zou, Carbon, 2012, 50, 2315-2321.

32 C. Tsouris, R. Mayes, J. Kiggans, K. Sharma, S. Yiacoumi, D. DePaoli and S. Dai, Environ. Sci. Technol., 2011, 45, 10243-10249.

33 H. Yin, S. Zhao, J. Wan, H. Tang, L. Chang, L. He, H. Zhao, Y. Gao and Z. Tang, Adv. Mater., 2013, 25, 6270-6276.

34 Z. Liu, G. Zhang, Z. Lu, X. Jin, Z. Chang and X. Sun, Nano Res., 2013, 6, 293-301.

35 B. Xu, D. Zheng, M. Jia, G. Cao and Y. Yang, Electrochim. Acta, 2013, 98, 176-182.

36 J. Deng, P. Ren, D. Deng and X. Bao, Angew. Chem., Int. Ed. Engl., 2015, 54, 2100-2104.

37 Y. Zhu, S. Murali, M. D. Stoller, K. J. Ganesh, W. Cai, P. J. Ferreira, A. Pirkle, R. M. Wallace, K. A. Cychosz, M. Thommes, D. Su, E. A. Stach and R. S. Ruoff, Science, 2011, 332, 1537-1541.

38 P. Xu, J. E. Drewes, D. Heil and G. Wang, Water Res., 2008, 42, 2605-2617.

39 C. J. Gabelich, T. D. Tran and A. I. H. M. Suffet, Environ. Sci. Technol., 2002, 36, 3010-3019.

40 C.-H. Hou and C.-Y. Huang, Desalination, 2013, 314, 124129.

41 J. Lee, S. Kim, C. Kim and J. Yoon, Energy Environ. Sci., 2014, 7, 3683-3689.

42 H. Jiang, J. Ma and C. Li, Adv. Mater., 2012, 24, 4197-4202.
43 Z.-Y. Yang, L.-J. Jin, G.-Q. Lu, Q.-Q. Xiao, Y.-X. Zhang, L. Jing, X.-X. Zhang, Y.-M. Yan and K.-N. Sun, Adv. Funct. Mater., 2014, 24, 3917-3925.

44 Y. Li, Z. Li and P. K. Shen, Adv. Mater., 2013, 25, 2474-2480. 45 S. Biniak, G. Szymanski, J. Siedlewski and A. Swiatkowski, Carbon, 1997, 35, 1799-1810.

46 D. Hulicova-Jurcakova, M. Seredych, G. Q. Lu and T. J. Bandosz, Adv. Funct. Mater., 2009, 19, 438-447.

47 H. Luo, Z. Liu, L. Chao, X. Wu, X. Lei, Z. Chang and X. Sun, J. Mater. Chem. A, 2015, 3, 3667-3675.

48 Z. Wen, X. Wang, S. Mao, Z. Bo, H. Kim, S. Cui, G. Lu, X. Feng and J. Chen, Adv. Mater., 2012, 24, 5610-5616.

49 Z. Liu, C. Zhang, L. Luo, Z. Chang and X. Sun, J. Mater. Chem., 2012, 22, 12149-12154.

50 J. Wang and S. Kaskel, J. Mater. Chem., 2012, 22, 2371023725.

51 X. Li, X. Zang, Z. Li, X. Li, P. Li, P. Sun, X. Lee, R. Zhang, Z. Huang, K. Wang, D. Wu, F. Kang and H. Zhu, Adv. Funct. Mater., 2013, 23, 4862-4869.

52 X. Fan, C. Yu, J. Yang, Z. Ling and J. Qiu, Carbon, 2014, 70, 130-141.

53 L. Zhao, L. Z. Fan, M. Q. Zhou, H. Guan, S. Y. Qiao, M. Antonietti and M. M. Titirici, Adv. Mater., 2010, 22, 5202-5206.

54 D. Hulicova-Jurcakova, M. Kodama, S. Shiraishi, H. Hatori, Z. H. Zhu and G. Q. Lu, Adv. Funct. Mater., 2009, 19, 18001809.

55 Z. Wen, X. Wang, S. Mao, Z. Bo, H. Kim, S. Cui, G. Lu, X. Feng and J. Chen, Adv. Mater., 2012, 24, 5610-5616.

56 M. Li, C. Liu, H. Cao, H. Zhao, Y. Zhang and Z. Fan, J. Mater. Chem. A, 2014, 2, 14844-14851.

57 N. P. Wickramaratne, J. Xu, M. Wang, L. Zhu, L. Dai and M. Jaroniec, Chem. Mater., 2014, 26, 2820-2828.

58 A. Sumboja, C. Y. Foo, X. Wang and P. S. Lee, Adv. Mater., 2013, 25, 2809-2815.

59 L. Dai, D. W. Chang, J. B. Baek and W. Lu, Small, 2012, 8, 1130-1166.

60 Y. Zhao, J. Liu, Y. Hu, H. Cheng, C. Hu, C. Jiang, L. Jiang, A. Cao and L. Qu, Adv. Mater., 2013, 25, 591-595.

61 H. D. J. Jiayan Luo and J. Huang, ACS Nano, 2013, 7, 14641471.

62 Q. Dong, G. Wang, B. Qian, C. Hu, Y. Wang and J. Qiu, Electrochim. Acta, 2014, 137, 388-394.

63 L. Zou, G. Morris and D. Qi, Desalination, 2008, 225, 329-340. 64 H. Kato, K. Asakura and A. A. Kudo, J. Am. Chem. Soc., 2003, 125, 3082-3089.

65 Y. Wang, R. Wang, S. Xu, Q. Liu and J. Wang, Desalin. Water Treat., 2014, 52, 1-9.

66 Z. Peng, D. Zhang, T. Yan, J. Zhang and L. Shi, Appl. Surf. Sci., 2013, 282, 965-973.

67 A. G. El-Deen, N. A. M. Barakat, K. A. Khalil, M. Motlak and H. Yong Kim, Ceram. Int., 2014, 40, 14627-14634.

68 H. Li, L. Zou, L. Pan and Z. Sun, Environ. Sci. Technol., 2010, 44, 8692-8697. 